\title{
Dynamical Behaviour of the Light Pulses through the Optical Fiber: Two Nonlinear Atangana Conformable Fractional Evolution Equations
}

\author{
S. H. Alfalqi, ${ }^{1}$ Mostafa M. A. Khater ${ }^{(D)},{ }^{2,3}$ J. F. Alzaidi, ${ }^{1}$ and Dianchen Lu $^{2}$ \\ ${ }^{1}$ Department of Mathematics, Faculty of Science and Arts, Mahayil Asir King Khalid University, Abha, Saudi Arabia \\ ${ }^{2}$ Department of Mathematics, Faculty of Science, Jiangsu University, Jiangsu 212013, China \\ ${ }^{3}$ Department of Mathematics, El Obour Institutes, Cairo 11828, Egypt
}

Correspondence should be addressed to Mostafa M. A. Khater; mostafa.khater2024@yahoo.com

Received 9 September 2020; Accepted 4 October 2020; Published 21 October 2020

Academic Editor: Hijaz Ahmad

Copyright (c) 2020 S. H. Alfalqi et al. This is an open access article distributed under the Creative Commons Attribution License, which permits unrestricted use, distribution, and reproduction in any medium, provided the original work is properly cited.

\begin{abstract}
This study, using the extended simplest method of equation, examines the explicit movement solutions of both the Schwarzian Korteweg-de Vries (SKdV) and (2+1)-Ablowitz-Kaup-Newell-Segur (AKNS.) equation. These models show the movement of the waves in optical fiber mathematically. The SKdV equation explains the movement of the isolated waves in diverse fields and on the site in a small space microsection. Some solutions obtained have been developed to show the physical and dynamic behaviors of these solutions in the obtained wave.
\end{abstract}

\section{Introduction}

Partial differential equations (PDEs) have been playing an essential role in describing and studying some complex phenomena in distinct branches of science [1-5]. These phenomena have been formulated in nonlinear PDEs with an integer order or fractional order [6-8]. Studying these mathematical models have been forcing many research groups in physics, chemistry, mathematics, and so on to derive practical and powerful computational schemes (analytical, semianalytical, and numerical techniques) for constructing exact and numerical solutions [9-15]. These schemes include the modified and generalized Kudryashov methods, the extended tanh-function method, the improved $\tan (\phi / 2)$ expansion method, the novel, improved, extended, and generalized $\left(G^{\prime} / G\right)$ expansion method, the extended and generalized $e^{-\phi(\xi)}$ expansion method, the Khater method, the modified Khater method, the Adomian decomposing method, the B-spline schemes, and so on [16-24].
In this research, we investigate two primary mathematical models in the optical fiber via the extended simplest equation method. The first model is Atangana conformable fractional SKdV equation that was derived by Krichever and Novikov in the following form [25]:

$$
\frac{\mathscr{D}_{t}^{q} \mathscr{U}}{\mathscr{U}_{x}}+\left(\frac{\mathscr{U}_{x x}}{\mathcal{U}_{x}}\right)_{x}-\frac{1}{2}\left(\frac{\mathscr{U}_{x x}}{\mathcal{U}_{x}}\right)^{2}=0, \quad(0<q<1),
$$

where $\mathscr{U}=\mathscr{U}(x, t)$ satisfies Newton's equation of motion in a cubic potential. Equation (1) is also given by [26]

$$
\begin{aligned}
& \mathscr{D}_{t}^{q} \mathscr{G}+\frac{1}{4} \mathscr{G}_{x x z}-\frac{\mathscr{G}_{x} \mathscr{G}_{x z}}{2 \mathscr{G}}-\frac{\mathscr{G}_{x x} \mathscr{G}_{z}}{4 \mathscr{G}}+\frac{\mathscr{G}_{x}^{2} \mathscr{G}_{z}}{2 \mathscr{G}^{2}} \\
& -\frac{\mathscr{G}_{x}}{8} \int\left(\frac{\mathscr{G}_{x}^{2}}{\mathscr{G}^{2}}\right)_{z} \mathrm{~d} x=0 .
\end{aligned}
$$

Equation (2) has an essential role in a right-moving soliton and the nonlocal form. However, we study a new form of equation (2) that is given in the following system [27]: 


$$
\left\{\begin{array}{l}
\mathscr{L}_{1} \mathscr{B}^{2} \mathscr{C}_{x}-\mathscr{L}_{1} \mathscr{B} \mathscr{B}_{x} C+\mathscr{B}^{2} \mathscr{C}_{x x z}-\mathscr{B}_{x x} \mathscr{B}_{z}-\mathscr{L}_{2} \mathscr{B}_{x} \mathscr{B}_{x z}+\mathscr{L}_{2} \mathscr{B}_{x}^{2} \mathscr{B}_{z}-\mathscr{B}^{4} \mathscr{B}_{z}=0 \\
\mathscr{D}_{t}^{q} \mathscr{B}-\mathscr{D}_{t}^{q} C=0
\end{array}\right.
$$

where $\mathscr{B}=\mathscr{B}(x, t), \mathscr{C}=\mathscr{C}(x, z, t)$. Additionally, $\mathscr{L}_{1}, \mathscr{L}_{2}$ are arbitrary constants. Using the following wave transformation

$\mathscr{B}(x, t)=\mathscr{B}(b), \mathscr{C}(x, z, t)=v(f), b=x+z-$

$(c / q)(t+1 / \Gamma(q))^{q}$ and then integrating the second equation of the transformed system once with zero constant of integration and substituting the result into the first equation of the same system lead to

$$
\mathscr{B}^{2} \mathscr{B}^{\prime \prime \prime}-\left(1+\mathscr{L}_{2}\right) \mathscr{B} \mathscr{B} \mathscr{B}^{\prime} \mathscr{B}^{\prime \prime}+\mathscr{L}_{2} \mathscr{B}^{3}-\mathscr{B}^{4} \mathscr{B}^{\prime}=0 .
$$

While the second model is the Atangana conformable fractional $(2+1)$ AKNS equation which is so close to the first model. This model is given by [28-30]

$$
4 \mathscr{D}_{t}^{q} Q_{x}+Q_{x x x z}+8 Q_{x z} Q_{x}+4 Q_{z} Q_{x x}=0,
$$

where $\mathbb{Q}=\mathbb{Q}(x, z, t)$. Applying the next wave transformation $\mathbb{Q}=\mathbb{Q}(b), b=x+z-(c / q)(t+(1 / \Gamma(q)))^{q}$ to equation (5) gives

$$
-4 c Q^{\prime}+Q^{\prime \prime \prime}+6 Q^{\prime 2}=0
$$

Balancing the terms in equations (4) and (6) based on the next principle of homogenous rule,

$$
\begin{gathered}
D\left[\frac{d^{\varepsilon} \mathscr{P}(b)}{d b^{\varepsilon}}\right]=N+\varepsilon, \\
D\left[\mathscr{P}^{\varepsilon}\left(\frac{d^{\varepsilon} \mathscr{P}(b)}{d \mathscr{P}^{\varepsilon}}\right)^{s}\right]=\varepsilon N+s(N+\varepsilon),
\end{gathered}
$$

leads to $N=1$.

The rest sections are order in the following order. Section 2 gives the implementation of the extended simplest equation method for the Atangana conformable fractional SKdV equation (8) and the Atangana conformable fractional $(2+1)$-AKNS equation (9). Also, some solutions are sketched to illustrate the physical behaviour of the wave solutions. Section 3 produces a conclusion of our paper.

\section{Application}

In this section, we apply the extended simplest equation method to the SKdV equation and the $(2+1)$-AKNS equation for constructing the exact traveling and solitary wave solutions.

2.1. The SKdVEquation. According to the extended simplest equation method and value of homogenous balance value, we get

$$
\mathscr{B}(b)=\sum_{i=-N}^{N} a_{i} f(b)^{i}=\frac{a_{-1}}{f(b)}+a_{0}+f(b) a_{1}
$$

where $a_{-1}, a_{0}$, and $a_{1}$ are arbitrary constants. Also, $f(b)$ follows the next ODE:

$$
f^{\prime}(b)=\alpha+\lambda f(b)+\mu f(b)^{2},
$$

where $\alpha, \lambda$, and $\mu$ are the arbitrary constants. Substituting equation (8) and its derivative along equation (9) into equation (4) and collecting all terms with the same power of $f^{i}(b), i=(0,1,2, \ldots)$ lead to a system of equations. Solving this system with any computer software yields family one:

$$
\begin{aligned}
a_{0} \longrightarrow & \frac{1}{2}\left(-\lambda-\sqrt{\lambda^{2}-4 \alpha \mu}\right), \quad a_{1} \longrightarrow 0, a_{-1} \longrightarrow \\
& -\alpha, \mathscr{L}_{2} \longrightarrow 3 .
\end{aligned}
$$

Subsequently, the explicit solutions of the fractional $\mathrm{SKdV}$ equation are given as follows.

When $\lambda=0, \alpha \mu<0$,

$$
\begin{aligned}
& \mathscr{B}_{1}(x, t)=-\sqrt{-\alpha \mu}+\sqrt{-\alpha \mu} \operatorname{Coth}\left[\mathscr{H} \sqrt{-\alpha \mu} \mp \frac{\log [\vartheta]}{2}\right], \\
& \mathscr{B}_{2}(x, t)=-\sqrt{-\alpha \mu}+\sqrt{-\alpha \mu} \operatorname{Tanh}\left[\mathscr{H} \sqrt{-\alpha \mu} \mp \frac{\log [\vartheta]}{2}\right] .
\end{aligned}
$$

When $4 \alpha \mu>\lambda^{2}$,

$$
\begin{aligned}
& \mathscr{B}_{3}(x, t)=-\frac{\lambda}{2}-\frac{1}{2} \sqrt{\lambda^{2}-4 \alpha \mu}+\frac{2 \alpha \mu}{\lambda-\sqrt{-\lambda^{2}+4 \alpha \mu \operatorname{Tan}\left[(1 / 2)(\mathscr{H}+9) \sqrt{-\lambda^{2}+4 \alpha \mu}\right]},} \\
& \mathscr{B}_{4}(x, t)=-\frac{\lambda}{2}-\frac{1}{2} \sqrt{\lambda^{2}-4 \alpha \mu}+\frac{2 \alpha \mu}{\lambda-\sqrt{-\lambda^{2}+4 \alpha \mu} \operatorname{Cot}\left[(1 / 2)(\mathscr{H}+\vartheta) \sqrt{-\lambda^{2}+4 \alpha \mu}\right]}, \\
& a_{0} \longrightarrow \frac{1}{2}\left(\lambda-\sqrt{\lambda^{2}-4 \alpha \mu}\right), \quad a_{1} \longrightarrow \mu, a_{-1} \longrightarrow 0, \mathscr{L}_{2} \longrightarrow 3 .
\end{aligned}
$$

where $\mathscr{H}=x+z-(c / q)(t+(1 / \Gamma(q)))^{q}$.

Family two: 


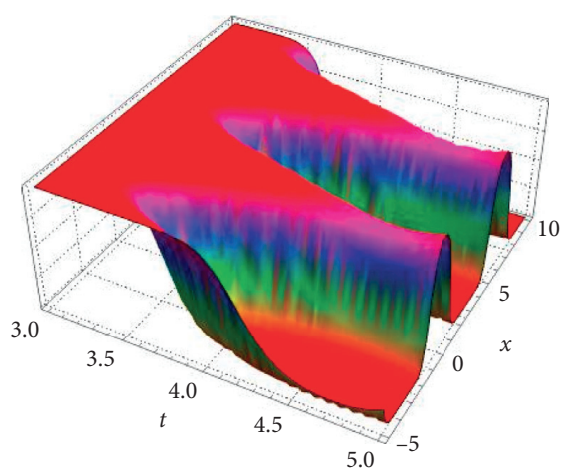

(a)

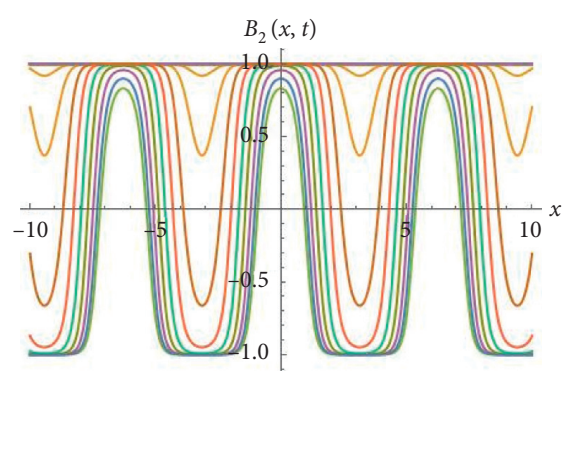

(b)

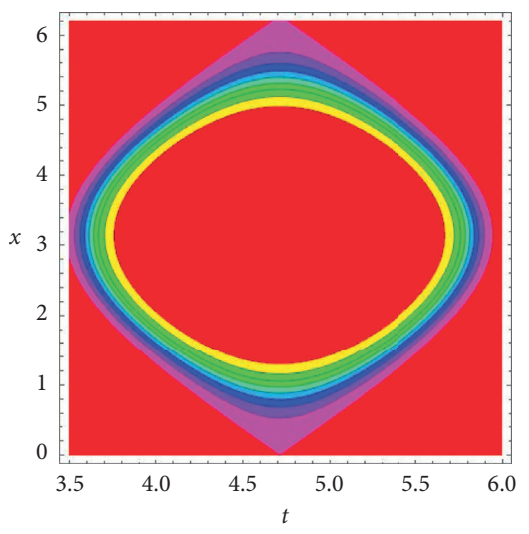

(c)

FIGURE 1: Periodic kink solitary wave solutions of equation (11) in three-dimensional, two-dimensional, and contour plot 3D for $\lambda=6, a_{1}=3, \mu=-9, c=-3$, and $\vartheta=2$.

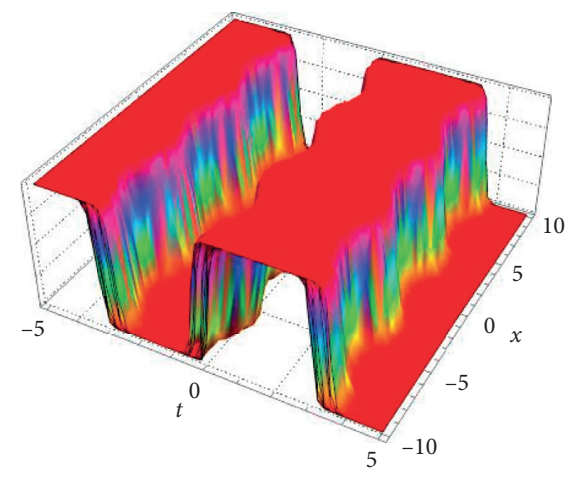

(a)

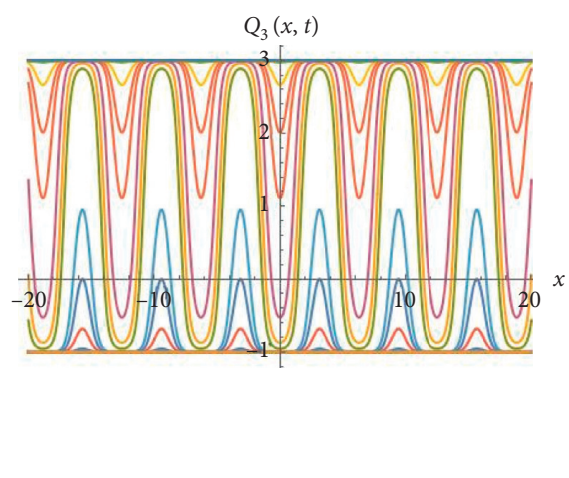

(b)

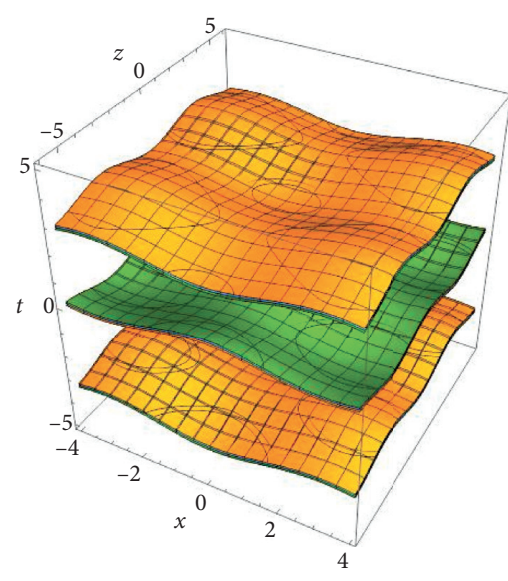

(c)

FIgURE 2: Kink solitary wave solutions of equation (21) in three-dimensional, two-dimensional and contour plot 3D for $a_{0}=1, \alpha=-1, \mu=4$, and $\vartheta=3$.

Subsequently, the explicit solutions of the fractional SKdV equation are given as follows.

When $\lambda=0, \alpha \mu<0$,

$$
\begin{aligned}
& \mathscr{B}_{5}(x, t)-\sqrt{-\alpha \mu}+\sqrt{-\alpha \mu} \operatorname{Tanh}\left[\mathscr{H} \sqrt{-\alpha \mu} \mp \frac{\log [\vartheta]}{2}\right], \\
& \mathscr{B}_{6}(x, t)=-\sqrt{-\alpha \mu}+\sqrt{-\alpha \mu} \operatorname{Coth}\left[\mathscr{H} \sqrt{-\alpha \mu} \mp \frac{\log [\vartheta]}{2}\right] .
\end{aligned}
$$

For $\lambda>0$,

$$
\mathscr{B}_{7}(x, t)=-\frac{\lambda}{2}-\frac{\sqrt{\lambda^{2}}}{2}+\frac{\lambda}{1-e^{(\mathscr{H}+9) \lambda} \mu} .
$$

For $\lambda<0$,

$$
\mathscr{B}_{8}(x, t)=\frac{\lambda}{2}-\frac{\sqrt{\lambda^{2}}}{2}-\mu+\frac{\mu}{1+e^{(\mathscr{H}+9) \lambda} \mu} .
$$

When $4 \alpha \mu>\lambda^{2}$,

When $\alpha=0$,

$$
\begin{gathered}
\mathscr{B}_{9}(x, t)=-\frac{1}{2} \sqrt{\lambda^{2}-4 \alpha \mu}+\frac{1}{2} \sqrt{-\lambda^{2}+4 \alpha \mu} \operatorname{Tan}\left[\frac{1}{2}(\mathscr{H}+\vartheta) \sqrt{-\lambda^{2}+4 \alpha \mu}\right], \\
\mathscr{B}_{10}(x, t)=-\frac{1}{2} \sqrt{\lambda^{2}-4 \alpha \mu}+\frac{1}{2} \sqrt{-\lambda^{2}+4 \alpha \mu} \operatorname{Cot}\left[\frac{1}{2}(\mathscr{H}+\vartheta) \sqrt{-\lambda^{2}+4 \alpha \mu}\right],
\end{gathered}
$$


where $\mathscr{H}=x+z-(c / q)(t+(q / \Gamma(q)))^{q}$

2.2. The $(2+1)-A K N S$ Equation. According to the extended simplest equation method and value of homogenous balance value, we get

$$
Q(b)=\sum_{i=-N}^{N} a_{i} f(b)^{i}=\frac{a_{-1}}{f(b)}+a_{0}+f(b) a_{1},
$$

where $a_{-1}, a_{0}$, and $a_{1}$ are the arbitrary constants. Also, $f(b)$ satisfies equation (9). Substituting equation (18) and its derivative along (9) into equation (6) and collecting all terms with the same power of $f^{i}(b), i=(0,1,2, \ldots)$ lead to a system of algebraic equations. Solving this system with any computer software yields

$$
a_{-1} \longrightarrow 0, a_{1} \longrightarrow-\mu, c \longrightarrow \frac{1}{4}\left(\lambda^{2}-4 \alpha \mu\right) .
$$

Subsequently, the explicit solutions of the fractional $(2+1)$-AKNS equation are given as follows.

When $\lambda=0$,

For $\alpha \mu>0$,

$$
\begin{aligned}
& Q_{1}(x, t)=a_{0}-\sqrt{\alpha \mu} \operatorname{Tan}\left[\sqrt{\alpha \mu}\left(x+z+\vartheta+\frac{\alpha \mu(t+(1 / \Gamma[q]))^{q}}{q}\right)\right], \\
& Q_{2}(x, t)=-\sqrt{\alpha \mu} \operatorname{Cot}\left[\sqrt{\alpha \mu}\left(x+z+\vartheta+\frac{\alpha \mu(t+(1 / \Gamma[q]))^{q}}{q}\right)\right]+a_{0} .
\end{aligned}
$$

For $\alpha \mu<0$,

$$
\begin{aligned}
& \mathscr{Q}_{3}(x, t)=a_{0}-\sqrt{-\alpha \mu} \operatorname{Tanh}\left[\sqrt{-\alpha \mu}\left(x+z+\frac{\alpha \mu(t+(1 / \Gamma[q]))^{q}}{q}\right) \mp \frac{\log [\vartheta]}{2}\right], \\
& \mathbb{Q}_{4}(x, t)=-\sqrt{-\alpha \mu} \operatorname{Coth}\left[\sqrt{-\alpha \mu}\left(x+z+\frac{\alpha \mu(t+(1 / \Gamma[q]))^{q}}{q}\right) \mp \frac{\log [\vartheta]}{2}\right]+a_{0} .
\end{aligned}
$$

When $\alpha=0$,

For $\lambda>0$,

$$
Q_{5}(x, t)=\lambda+\frac{\lambda}{-1+e^{\lambda\left(x+z+9-\left(\lambda^{2}(t+(1 / \Gamma[q]))^{q} / 4 q\right)\right)} \mu}+a_{0} .
$$

For $\lambda<0$,

$$
\mathbb{Q}_{6}(x, t)=\mu-\frac{\mu}{1+e^{\lambda\left(x+z+9-\left(\lambda^{2}(t+(1 / \Gamma[q]))^{q} / 4 q\right)\right) \mu+a_{0}} .}
$$

When $4 \alpha \mu>\lambda^{2}$,

$$
\begin{aligned}
& \mathbb{Q}_{7}(x, t)=\frac{\lambda}{2}+a_{0}-\frac{1}{2} \sqrt{-\lambda^{2}+4 \alpha \mu} \operatorname{Tan}\left[\frac{1}{2} \sqrt{-\lambda^{2}+4 \alpha \mu}\left(x+z+\vartheta-\frac{\left(\lambda^{2}-4 \alpha \mu\right)(t+(1 / \Gamma[q]))^{q}}{4 q}\right)\right], \\
& Q_{8}(x, t)=\frac{\lambda}{2}-\frac{1}{2} \sqrt{-\lambda^{2}+4 \alpha \mu} \operatorname{Cot}\left[\frac{1}{2} \sqrt{-\lambda^{2}+4 \alpha \mu}\left(x+z+\vartheta-\frac{\left(\lambda^{2}-4 \alpha \mu\right)(t+(1 / \Gamma[q]))^{q}}{4 q}\right)\right]+a_{0} .
\end{aligned}
$$

\section{Conclusion}

This paper has investigated the exact traveling and solitary wave solutions of the fractional SKdV equation and the fractional $(2+1)$-AKNS equation. The extended simplest equation method has successfully been implemented and some new distinct optical solitary wave solutions are obtained for both models. Some solutions have been sketched in three types (three-dimensional, two-dimensional, and contour plots) (Figures 1 and 2). The powerful effect of the used method is illustrated. Moreover, the ability of applying to different types of nonlinear evolution equations has been verified.

\section{Data Availability}

The data that support the findings of this study are available from the corresponding author (Mostafa M. A. Khater) upon reasonable request.

\section{Conflicts of Interest}

The authors declare that there are no conflicts of interest.

\section{Authors' Contributions}

All the authors conceived the study, participated in its design and coordination, drafted the manuscript, participated in the sequence alignment, and read and approved the final manuscript.

\section{Acknowledgments}

The authors are thankful to the Deanship of Scientific Research at the King Khalid University, Abha, KSA, for funding this work through research group under grant number (R.G.P-1/151/40). 


\section{References}

[1] A. H. Abdel-Aty, M. M. Khater, D. Baleanu, E. M. Khalil, J. Bouslimi, and M. Omri, "Abundant distinct types of solutions for the nervous biological fractional Fitz Hugh-Nagumo equation via three different sorts of schemes," Advances in Difference Equations, vol. 2020, no. 1, 17 pages, 2020.

[2] M. M. Khater and D. Baleanu, "On abundant new solutions of two fractional complex models," Advances in Difference Equations, vol. 2020, no. 1, 14 pages, 2020.

[3] H. Günerhan, F. S. Khodadad, H. Rezazadeh, and M. M. A. Khater, "Exact optical solutions of the $(2+1)$ dimensions Kundu-Mukherjee-Naskar model via the new extended direct algebraic method," Modern Physics Letters B, vol. 34, no. 22, Article ID 2050225, 2020.

[4] A.-H. Abdel-Aty, M. M. A. Khater, H. Dutta, J. Bouslimi, and M. Omri, "Computational solutions of the HIV-1 infection of CD4+ T-cells fractional mathematical model that causes acquired immunodeficiency syndrome (AIDS) with the effect of antiviral drug therapy," Chaos, Solitons \& Fractals, vol. 139, Article ID 110092, 2020.

[5] M. M. Khater, B. Ghanbari, K. S. Nisar, and D. Kumar, "Novel exact solutions of the fractional Bogoyavlensky-Konopelchenko equation involving the AtanganaBaleanu-Riemann derivative," Alexandria Engineering Journal, vol. 59, no. 5, 2020.

[6] D. Lu, K. U. Tariq, M. S. Osman, D. Baleanu, M. Younis, and M. M. A. Khater, "New analytical wave structures for the $(3+1)$-dimensional Kadomtsev-Petviashvili and the generalized Boussinesq models and their applications," Results in Physics, vol. 14, Article ID 102491, 2019.

[7] D. Lu, A. R. Seadawy, and M. M. A. Khater, "Structures of exact and solitary optical solutions for the higher-order nonlinear Schrödinger equation and its applications in monomode optical fibers," Modern Physics Letters B, vol. 33, no. 23, Article ID 1950279, 2019.

[8] M. S. Osman, D. Lu, and M. M. A. Khater, "A study of optical wave propagation in the nonautonomous Schrödinger-Hirota equation with power-law nonlinearity," Results in Physics, vol. 13, Article ID 102157, 2019.

[9] S. H. Alfalqi, J. F. Alzaidi, D. Lu, and M. Khater, "On exact and approximate solutions of (2+1)-dimensional KonopelchenkoDubrovsky equation via modified simplest equation and cubic B-spline schemes," Thermal Science, vol. 23, no. 6, p. 349, 2019.

[10] H. A. Suleman, F. A. Jameel, L. U. Dianchen, and M. M. Khater, "ON exact and approximate solutions OF $(2+$ 1)-D konopelchenko-dubrovsky equation via modified simplest equation And CUBIC B-spline schemes," Thermal Science, vol. 23, no. 6, pp. S1889-S1899, 2019.

[11] X. Zhang, Y. Wu, and L. Caccetta, "Nonlocal fractional order differential equations with changing-sign singular perturbation," Applied Mathematical Modelling, vol. 39, no. 21, pp. 6543-6552, 2015.

[12] X. Zhang, L. Liu, and Y. Wu, "Multiple positive solutions of a singular fractional differential equation with negatively perturbed term," Mathematical and Computer Modelling, vol. 55, no. 3-4, pp. 1263-1274, 2012

[13] X. Zong and F. Wu, "Exponential stability of the exact and numerical solutions for neutral stochastic delay differential equations," Applied Mathematical Modelling, vol. 40, no. 1, pp. 19-30, 2016.
[14] A. Jannelli, M. Ruggieri, and M. Paola Speciale, "Exact and numerical solutions of time-fractional advection-diffusion equation with a nonlinear source term by means of the Lie symmetries," Nonlinear Dynamics, vol. 92, no. 2, pp. 543-555, 2018.

[15] Khodadad, F. Samsami, F. Nazari, M. Eslami, and H. Rezazadeh, "Soliton solutions of the conformable fractional Zakharov-Kuznetsov equation with dual-power law nonlinearity," Optical and Quantum Electronics, vol. 49, no. 11, p. 384, 2017.

[16] P. Guo and C. J. Li, "Razumikhin-type theorems on the moment stability of the exact and numerical solutions for the stochastic pantograph differential equations," Journal of Computational and Applied Mathematics, vol. 355, no. 1, pp. 77-90, 2019.

[17] P. Guo and C.-J. Li, "Razumikhin-type technique on stability of exact and numerical solutions for the nonlinear stochastic pantograph differential equations," BIT Numerical Mathematics, vol. 59, no. 1, pp. 77-96, 2019.

[18] R. Cherniha, K. Gozak, and J. Waniewski, "Exact and numerical solutions of a spatially-distributed mathematical model for fluid and solute transport in peritoneal dialysis," Symmetry, vol. 8, no. 6, p. 50, 2016.

[19] D. Lu, A. R. Seadawy, and M. M. Khater, "Structure of solitary wave solutions of the nonlinear complex fractional generalized Zakharov dynamical system," Advances in Difference Equations, vol. 2018, no. 1, p. 266, 2018.

[20] M. M Khater, "Comment on four papers of Elsayed ME Zayed, Abdul-Ghani Al-Nowehy, Reham MA Shohib and Khaled AE Alurrfi (Optik 130 (2017) 1295-1311 \& Optik 143 (2017) 84-103 \& Optik 158 (2018) 970-984 \& optik 144 (2017) 132-148)," Optik, vol. 172, pp. 585-587, 2018.

[21] A. R. Seadawy, L. Dianchen, and M. M. Khater, "Reply of the manuscript of authors (Elsayed and Abdul-Ghani) in title (Comment on the paper of our paper [Superlattices and Microstructures, 113 (2018) 346-358])," Superlattices and Microstructures, vol. 123, pp. 460-464. In press, 2018.

[22] A. R. Seadawy, D. Lu, and M. M. Khater, "Structure of optical soliton solutions for the generalized higher-order nonlinear Schrödinger equation with light-wave promulgation in an optical fiber," Optical and Quantum Electronics, vol. 50, no. 9, p. 333, 2018.

[23] A. Benslimane, S. Bouzidi, and M. Methia, "Displacements and stresses in pressurized thick-walled FGM cylinders: exact and numerical solutions," International Journal of Pressure Vessels and Piping, vol. 168, pp. 219-224, 2018.

[24] P. Guo and C. J. Li, "Almost sure stability with general decay rate of exact and numerical solutions for stochastic pantograph differential equations," Numerical Algorithms, vol. 80, no. 4, pp. 1391-1411, 2019.

[25] M. Euler and N. Euler, "On Möbius-invariant and symmetryintegrable evolution equations and the Schwarzian derivative," Studies in Applied Mathematics, vol. 143, no. 2, pp. 139-156, 2019.

[26] M. M. A. Khater, A. R. Seadawy, and D. Lu, "Bifurcations of solitary wave solutions for (two and three)-dimensional nonlinear partial differential equation in quantum and magnetized plasma by using two different methods," Results in Physics, vol. 9, pp. 142-150, 2018.

[27] Z. Li, "Diversity soliton excitations for the (2+1)-dimensional Schwarzian Korteweg-de Vries equation," Thermal Science, vol. 22, no. 4, pp. 1781-1786, 2018.

[28] W. Gao, G. Yel, H. Mehmet Baskonus, and C. Cattani, "Complex solitons in the conformable $(2+1)$-dimensional 
Ablowitz-Kaup-Newell-Segur equation," AIMS Mathematics, vol. 5, no. 1, p. 86, 2019.

[29] A. Issasfa and J. Lin, "Lump and mixed rogue-soliton solutions to the 2+1 dimensional Ablowitz-Kaup-Newell-Segur equation," Journal of Applied Analysis and Computation, vol. 10, no. 1, pp. 314-325, 2019.

[30] G. Xue, Q. Zhou, A. Biswas, A. Kamis Alzahrani, and W. Liu, "Darboux transformation for a generalized Ablowitz-KaupNewell-Segur hierarchy equation," Physics Letters A, vol. 384, Article ID 126394, 2020. 